\title{
Strength-based Signal Detection in PAM OOK Concentration-Encoded Molecular Communication
}

\author{
Mohammad Upal Mahfuz \\ University of Wisconsin-Green Bay \\ 2420 Nicolet Drive, Green Bay, \\ Wisconsin, 54311, USA \\ +1 (920) 465-2790 \\ mahfuzm@uwgb.edu
}

\begin{abstract}
In this paper, the expressions of achievable strength-based suboptimum detection probabilities of concentration-encoded molecular communication (CEMC) system have been derived based on pulse-amplitude modulated (PAM) on-off keying (OOK) modulation scheme. Results show that the detection performance of a PAM OOK CEMC system significantly depends on the statistical parameters of diffusion noise and intersymbol interference (ISI). Analytical performances of ISI-free and ISIaffected scenarios have been explained and compared based on receiver operating characteristics (ROC) curves for impulse (i.e. spike)-modulated (IM) and PAM schemes. It is shown that the effects of diffusion noise and ISI on ROC can be explained separately based on their communication range-dependent statistics.
\end{abstract}

\section{Categories and Subject Descriptors}

H.1.1 [Systems and Information Theory]: Information theory

\section{General Terms}

Theory

\section{Keywords}

Molecular communication, concentration-encoding, strength-based detection, pulse transmission, nanonetworks.

\section{INTRODUCTION}

Concentration-encoded molecular communication (CEMC) is an information encoding technique that relies on transmitting a single type of information molecules and varying the number of transmitted molecules in order to materialize molecular communication (MC) among bio-nanomachines [1, 2]. Pulse amplitude modulated (PAM) on-off keying (OOK) CEMC system is a common and popular form of $\mathrm{MC}$ among natural bionanomachines where a transmitting nanomachine $(\mathrm{TN})$ releases a pulse of molecules in the form of a fixed number of molecules per second (s) for a finite length pulse-width $T_{p}>0$ when the TN wants to send bit 1 and it does not release any molecule at all when it wants to send bit 0 [1]. Correspondingly, in strength-based detection, a receiving nanomachine ( $\mathrm{RN})$ observes signal strength [3], i.e. the number of molecules available to $\mathrm{RN}$ during the symbol duration and compares it to a threshold. In impulse modulation
(IM), the TN releases all the molecules at a time and so ideally $T_{p}$ $=0$. However, in PAM, $0<T_{p} \leq T_{\text {sym }}$ where $T_{\text {sym }}$ denotes the symbol interval. When $T_{p}=T_{s y m}$, it means that TN remains "on" in the entire $T_{\text {sym }}$ in order to send bit 1 and "off" otherwise, noting that 0 $<T_{p}<T_{\text {sym }}$ indicates a pulse-transmitted signal where the TN remains "on" for a period less than the symbol interval of the system. The quantity $\alpha=T_{p} / T_{\text {sym }}$ is considered as the duty cycle of the transmitter system: $\alpha=0$ (ideal case, e.g. IM) and $\alpha=1$ (practical case, e.g. PAM with $T_{p}=T_{\text {sym }}$ ). Here, $\alpha$ varying from 0 to 1 denotes the percentage of the symbol interval being used by the $\mathrm{TN}$ in releasing the information molecules to represent bit 1. Given a fixed number of molecules $A_{M o}$ to be released in order to represent bit 1 , when $0<T_{p} \leq T_{s y m}$, the TN adjusts the number of released molecules to $A_{M o} / T_{p}$ over the pulse width $T_{p}$. Although PAM OOK with $0<\alpha \leq 1$ has been identified as a popular scheme for CEMC so far [1], its detection capability is still unexplored. While several relevant works [4-6] have investigated the performance of PAM OOK CEMC system and its dependence on communication range and transmission data rate, to the best of the author's knowledge, none of the available open literature has addressed the issue of achievable detection capability of PAM OOK scheme in CEMC. In this paper, the following contributions have been made:

- First, the analytical expressions of simplistic strength-based suboptimum detection probability $P_{D}$ in the presence of diffusion-noise with and without the effects of ISI have been derived.

- Second, the statistics of diffusion-based noise and intersymbol interference (ISI) for PAM OOK CEMC system have been derived. It is found that the dependence of diffusion-noise and ISI separately on the desired signal makes it extremely challenging to detect the desired signal correctly.

- Finally, the effects of diffusion noise and ISI on $P_{D}$ have been separated and the detection performance have been explained when communication range and transmission data rate vary.

This paper is organized as follows: Section 2 presents the system model. Section 3 provides the detection model with and without the effects of ISI at the RN. Section 4 derives the statistics of diffusion noise and ISI in the case of PAM OOK receiver in CEMC. Key findings obtained through numerical results have been provided in Section 5. Finally, Section 6 summarizes the importance of the results and concludes the paper.

\section{SYSTEM MODEL}

We consider a pair of nanomachines, namely $\mathrm{TN}$ and $\mathrm{RN}$, in an unbounded three-dimensional propagation medium [1]. TN and RN are respectively located at the origin $(0,0,0)$ and any location represented by the vector $\vec{r}=(x, y, z)$ where $r^{2}=x^{2}+y^{2}+z^{2}$. TN 
and $\mathrm{RN}$ are assumed to be synchronized in time, which can be made possible by using external signals [4]. As shown in Fig. 1, the TN transmits molecules in a time-slotted manner, meaning that, when $\alpha=1$, to send a bit 1 , TN transmits molecules at a fixed rate of $A=A_{M o} / T_{\text {sym }}$ molecules/s during the entire $T_{\text {sym }}$ and it does not send any molecules when it wants to send a bit 0 . When $\alpha=0$, the TN transmits all the $A_{M o}$ molecules at a time in an impulsive (i.e. spike) fashion, e.g. in IM.

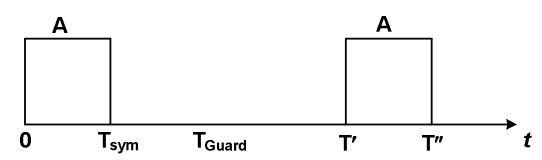

Fig. 1 Pulse-transmitted OOK signaling: $T^{\prime}=T_{s y m}+T_{\text {Guard }}$ and $T^{\prime \prime}=2 T_{\text {sym }}+T_{\text {Guard }}$. Here $T_{\text {Guard }}$ is the guard time between successive symbols.

Since information molecules undergo ideal diffusion-based propagation [5], the number of molecules available at the $\mathrm{RN}$ is a random variable due to effects of both diffusion-based noise and ISI [6]. The RN senses the occupancy of its receptors at uniform sampling intervals of $t_{\mathrm{s}}=1 \mathrm{~s}$. In order to ensure statistically independent, uncorrelated samples of concentration intensity, $t_{\mathrm{s}}$ needs to be reasonably larger than the waiting time of a molecule in the receiver sensing volume [9]. While uniform sampling is adopted in this paper, other sampling techniques, e.g. nonuniform sampling, may also be used in such cases.

\section{DETECTION MODEL}

\subsection{ISI-free Detection}

Consider that signal detection model can be expressed in terms of hypotheses $\mathrm{H}_{0}$ and $\mathrm{H}_{1}$ when the TN sends bits 0 and 1 respectively as shown next. First, we consider the absence of ISI. For example, for the $i^{\text {th }}$ symbol, this can be realized in two ways, namely, either by assuming $T_{\text {Guard }}=\infty$ between two pulses corresponding to the two symbols, or by assuming all the previous bits $b_{j}, j \in\{1,2, \ldots$, $(i-1)\}$, being 0 s so that they produce zero ISI at the RN respectively. In reality, $T_{\text {Guard }} \gg 10 \tau_{D S}$, where $\tau_{D S}$ is the root mean square (RMS) delay spread of CEMC channel [7]. The number of molecules available at the $\mathrm{RN}$ at a given time follows binomial distribution [6], which can be approximated to a normal distribution in CEMC [6]. The strength-based signal detection model can be expressed as below [9].

$$
z_{E D}=\left\{\begin{array}{cc}
\mathcal{N}\left(s_{E D}, \sigma_{S(E D)}^{2}\right) ; & \mathrm{H}_{1} \\
0 ; & \mathrm{H}_{0}
\end{array}\right.
$$

This yields the probabilities of false alarm $\left(P_{F A}\right)$ and detection $\left(P_{D}\right)$ as:

$$
\begin{gathered}
P_{F A}=\operatorname{Pr}\left\{z_{E D}>0 ; \mathrm{H}_{0}\right\}=0 \\
P_{D}=\operatorname{Pr}\left\{z_{E D}>0 ; \mathrm{H}_{1}\right\}=1-Q\left(s_{E D} / \sigma_{s(E D)}\right)=1-Q(\Lambda)
\end{gathered}
$$

Here $s_{E D}$ denotes the mean number of desired molecular signal strength, $\sigma_{s(E D)}$ is the standard deviation of signal strength, and $Q$ (.) denotes the right-tail probability and can be expressed as $Q(\zeta)=\int_{\zeta}^{\infty}(1 / \sqrt{2 \pi}) \exp \left(-x^{2} / 2\right) d x$ and $Q^{-1}($.$) is the inverse of$ $Q($.$) [8]. Hence \Lambda=s_{E D} / \sigma_{s(E D)}$ denotes the desired signal to diffusion noise strength ratio, or alternatively, signal to noise ratio (SNR) at the RN.

\subsection{ISI-affected Detection}

When either $10 \tau_{D S} \geq T_{\text {Guard }} \geq 0$ or at least one of the previously transmitted bits $b_{j}, j \in\{1,2, \ldots,(i-1)\}$ is a bit 1 , ISI-producing molecules will impact the signal strength of the $i^{\text {th }}$ symbol at the RN. For example, when $T_{\text {Guard }}=0$, TN sends pulses at every $T_{\text {sym }}$ seconds. With the effects of ISI, the PAM OOK signal detection model can be expressed as below.

$$
z_{E D}=\left\{\begin{array}{cc}
\mathcal{N}\left(s_{E D}+\mu_{I S I(E D)}, \sigma_{S(E D)}^{2}+\sigma_{I S I(E D)}^{2}\right) ; & \mathrm{H}_{1} \\
\mathcal{N}\left(\mu_{I S I(E D)}, \sigma_{I S I(E D)}^{2}\right) ; & \mathrm{H}_{0}
\end{array}\right.
$$

Here, $\mu_{I S I(E D)}$ and $\sigma_{I S I(E D)}^{2}$ denote the mean and variance of ISIproducing molecular strength respectively. Suboptimum receiver operating characteristics (ROC) curves can be obtained as below in terms of $P_{F A}$ and $P_{D}[8]$.

$$
\begin{aligned}
& P_{F A}=\operatorname{Pr}\left\{z_{E D}>\gamma ; \mathrm{H}_{0}\right\}=Q\left(\frac{\gamma-\mu_{I S I}}{\sigma_{I S I}}\right) \\
& P_{D}=\operatorname{Pr}\left\{z_{E D}>\gamma ; \mathrm{H}_{1}\right\}=Q\left(\frac{Q^{-1}\left(P_{F A}\right) R_{\sigma}-\Lambda}{\sqrt{1+R_{\sigma}^{2}}}\right)
\end{aligned}
$$

Here $\gamma$ denotes a threshold that can be found for a given $P_{F A}$. Unlike Eq. (2), Eq. (4) shows that in addition to $\Lambda$, in ISI-affected scenario, the detection performance is also a function of $P_{F A}$ and $R_{\sigma}=\sigma_{I S I(E D)} / \sigma_{S(E D)}$, i.e. the ratio of standard deviations of ISI strength to desired signal strength. Here, $R_{\sigma}$ bears the characteristics of the ISI that impact $P_{D}$ at the current symbol. Here $R_{\mu}=\mu_{I S I(E D)} / s_{E D}$, i.e. the ratio of mean values of ISI strength to desired signal strength.

Note that the ROC as shown in Eq. (4) does not depend on $\mu_{I S I(E D)}$. Note also that Eq. (4) shows the impact of ISI in $P_{D}$ such that plugging $R_{\sigma}=0$ into Eq. (4) yields the ISI-free detection as shown in Eq. (2).

Let us now determine the worst case ISI-affected scenario as follows. For the $i^{\text {th }}$ symbol, the worst-case detection would take place when all of the previous $(i-1)$ bits are $1 \mathrm{~s}$, hence contributing maximum to the ISI at the detection of the current bit. Therefore, in the worst-case scenario, the respective ISI quantities can be expressed as

$$
\begin{aligned}
& \mu_{I S I(E D)}^{(W C)}=\sum_{j=1}^{M} \mu_{I S L(E D)}(j) \\
& \sigma_{I S I(E D)}^{2(W C)}=\sum_{j=1}^{M} \sigma_{I S I(E D)}^{2}(j)
\end{aligned}
$$

where $M$ is the total number of previous bits considered (i.e. memory). For instance, for the $i^{- \text {th }}$ bit at the worst case, $M=(i-1)$.

Similarly, in the worst-case scenario, $R_{\sigma}^{(W C)}=\sigma_{I S I(E D)}^{(W C)} / \sigma_{S(E D)}$ where the superscript $(W C)$ denotes the worst-case scenario. Therefore, the worst-case ROC can be found as

$$
P_{D}^{(W C)}=Q\left(\frac{Q^{-1}\left(P_{F A}\right) R_{\sigma}^{(W C)}-\Lambda}{\sqrt{1+R_{\sigma}^{2(W C)}}}\right)
$$

Though $s_{E D}$ and $\sigma_{S(E D)}$ are independent of ISI, all of $s_{E D}, \sigma_{S(E D)}$, and $R_{\sigma}^{(W C)}$ depend on $r$ and $T_{s y m}$ and hence the necessity to find communication range- and rate-dependent characteristics of $P_{D}$. For a given $P_{F A}$, since a high $P_{D}$ provides a low BER, useful variations in the detection performance can be observed by obtaining the quantities $R_{\sigma}, R_{\sigma}^{(W C)}$, and $\Lambda$ numerically, and 
plugging them in Eqs. (2), (4), and (6) in order to find $P_{D}$ and $P_{D}^{(W C)}$ as shown next.

\section{DIFFUSION-BASED NOISE AND ISI STATISTICS}

In the analysis to follow, we show that, in a PAM OOK CEMC system, the signal strength can be expressed in terms of the impulse response of the channel. When the TN transmits $A_{M o} \gg 1$ molecules in an impulsive manner, the mean number of molecules (i.e. concentration) at the RN can be expressed as

$$
s(t)=A_{M o} p(t)
$$

where as shown below $p(t)$ is the probability of finding one molecule at time instant $t$ at the virtual receiving volume (VRV) surrounding the RN [6] and $d V=d x d y d z$ is the differential volume in VRV [6].

$$
p(t)=\iiint_{d V} \frac{1}{(4 \pi D t)^{3 / 2}} \exp \left(-\frac{x^{2}+y^{2}+z^{2}}{4 D t}\right) d x d y d z
$$

Mean concentration of information molecules at the $\mathrm{RN}$ due to PAM OOK system can be found by taking integral of the channel output due to impulsive transmission [5]. Therefore, using Eq. (7), with $t_{\mathrm{s}}$ as the sampling interval, yields the mean and the variance of the desired signal strength as below

$$
\begin{gathered}
s_{E D}=\sum_{n=0}^{N_{\text {samp }}} \sum_{i=0}^{n} s\left(i t_{s}\right) t_{s} \\
\sigma_{S(E D)}^{2}=\sum_{n=0}^{N_{\text {samp }}} \sum_{i=0}^{n} s\left(i t_{s}\right)\left(1-p\left(i t_{s}\right)\right) t_{s}^{2}
\end{gathered}
$$

where $i, n$ are temporal indices ${ }^{1}, N_{\text {samp }}$ is the total number of samples taken in each symbol, and $t_{s}=1 \mathrm{~s}$ is the sampling interval. Similarly, at the $i^{\text {th }}$ symbol, the mean and the variance of ISIproducing molecules originated in the $j^{\text {th }}$ symbol, $j \in\{1,2, \ldots$, $(i-1)\}$, can be expressed as below, where $k, n$ are temporal indices.

$$
\begin{gathered}
\mu_{I S I(E D)}(j)=\sum_{n=(i-j) N_{\text {samp }}+1}\left\{\sum_{k=0}^{n} s\left(k t_{s}\right)-\sum_{k=0}^{n-N_{\text {samp }}-1} s\left(k t_{s}\right)\right\} \\
\sigma_{I S I(E D)}^{2}(j)=\sum_{n=(i-j) N_{\text {samp }}+1}^{(i-j+1) N_{\text {samp }}}\left\{\sum_{k=0}^{n} s\left(k t_{s}\right)\left(1-p\left(k t_{s}\right)\right)-\sum_{k=0}^{n-N_{\text {samp }}-1} s\left(k t_{s}\right)\left(1-p\left(k t_{s}\right)\right.\right.
\end{gathered}
$$

As shown in Eq. (10), the quantities $\mu_{I S I(E D)}(j)$ and $\sigma_{I S I(E D)}^{2}(j)$ can be found by using $s(t)$ and $p(t)$ functions. In numerical simulations, $A_{M_{o}}=1000$ has been assumed.

\section{RESULTS}

In the absence of ISI, $P_{D}$ depends on $\Lambda$ only; however, when ISI is present, $P_{D}$ degrades and significantly depends on $P_{F A}, \Lambda$, and $R_{\sigma}$. Numerical simulations have been performed in order to find $\Lambda$ and $R_{\sigma}$ when TN and RN are spatially apart by $r$ and transmission data rate is given by $\Omega=1 / T_{\text {sym. }}$. In this paper, we provide the results of PAM scheme with $\alpha=1$, meaning that $T_{p}=T_{\text {sym }}$ in all the results reported in this paper. In the absence of ISI, Eq. (2) yields the BER performance as shown in Fig. 2 where $P_{D}$ and BER depends on $\Lambda$ only. When SNR is low, BER remains constant at 0.25 ; however, as SNR increases, BER decreases.

Figures 3(a) and 3(b) show the variations of SNR and ISI-related quantities $R_{\mu}$ and $R_{\sigma}$ when $r$ varies. As shown in Fig, 3(a), the IM

${ }^{1}$ Strength-based detection in CEMC is alternatively known as energy detection (ED) and hence the subscript " $E D$ ". scheme provides more SNR than the PAM scheme consistently for $r$ in the range from $400 \mathrm{~nm}$ up to more than $100 \mu \mathrm{m}$, which provides better detection probability as shown in Fig. 4. As shown in Fig. 3(b), both $R_{\mu}$ and $R_{\sigma}$ in the PAM scheme are higher than the corresponding values in the case of IM scheme, which explains the fact that the PAM scheme is more affected by the ISI-producing molecules than the IM scheme. This can be explained by Eq. (6) such that $P_{D}$ decreases as $R_{\sigma}$ increases. Note that, in the absence of ISI, $R_{\mu}=0$ and $R_{\sigma}=0$, and plugging these values in Eq. (6) yields Eq. (2) as derived earlier.

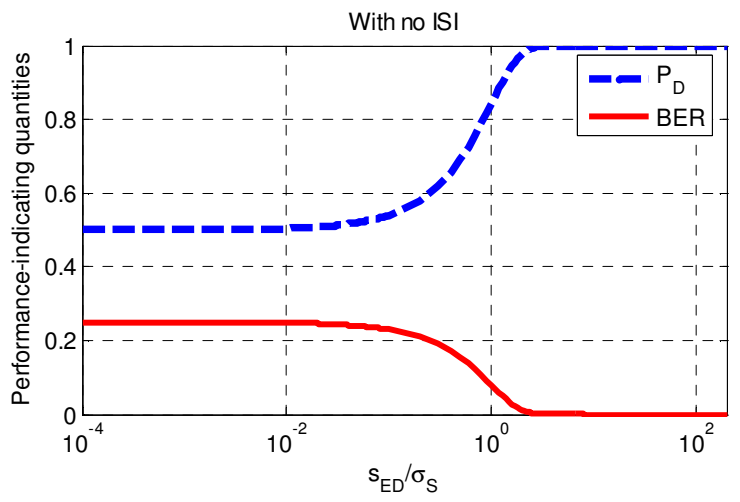

Fig. 2. Detection probability and BER in the absence of ISI

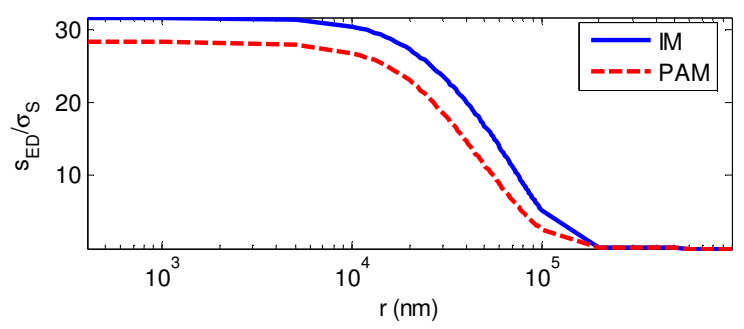

(a) SNR
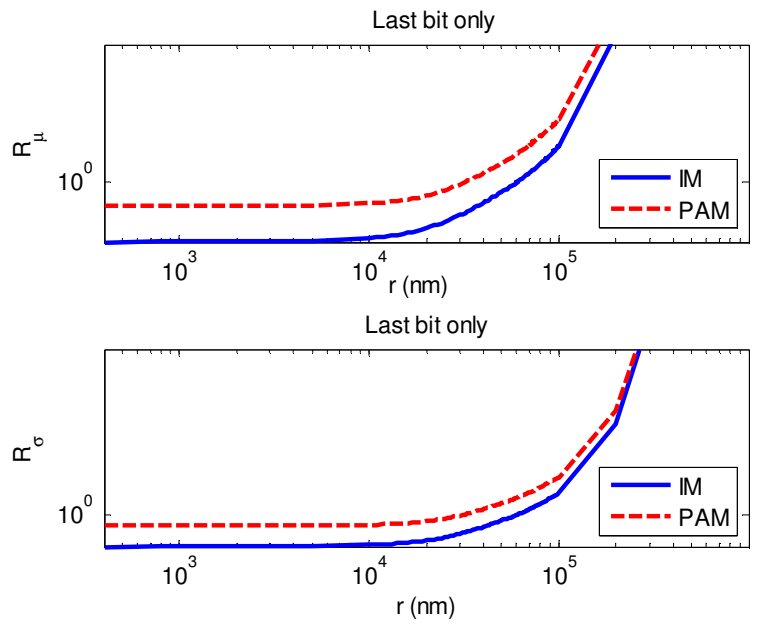

(b) ISI characteristics

Fig. 3. Diffusion noise characteristics in the presence of ISI 
Figure 4 shows the characteristics of detection probability when SNR varies in last-bit ISI (Figs. 4(a) and 4(b)) and worst-case ISI (Figs. 4(c) and 4(d)) scenarios. Comparing Figs. 4(a) with 4(b) shows that the PAM scheme performs worse than the IM scheme in the last-bit ISI scenario. Comparing Figs. 4(c) with 4(d) shows that both the IM and PAM schemes provide almost the same performance in the worst-case ISI scenario, the most effective reason behind this being the high number of ISI-producing molecules present in the worst-case scenario with both schemes.

\section{CONCLUSION}

In this paper, performance of a strength-based suboptimum receiver has been presented for the PAM scheme with $\alpha=1$ in CEMC system. In addition, the performance of the PAM scheme is further compared with that of the IM scheme. The analytical results and numerical simulations show that the PAM scheme with $\alpha=1$ is more affected by the ISI-producing molecules and hence the lower $P_{D}$ values when compared to the IM scheme under the same scenario. However, in the worst-case scenario when all the previous bits are 1s, both IM and PAM systems are equally affected by the ISI-producing molecules and so yield almost the same worst-case ISI performances. Future works of this research include an analysis of the PAM system when $0<\alpha \leq 1$ and transmission data rate varies.

\section{REFERENCES}

[1] M. U. Mahfuz, D. Makrakis, and H. T. Mouftah, "On the characterization of binary concentration-encoded molecular

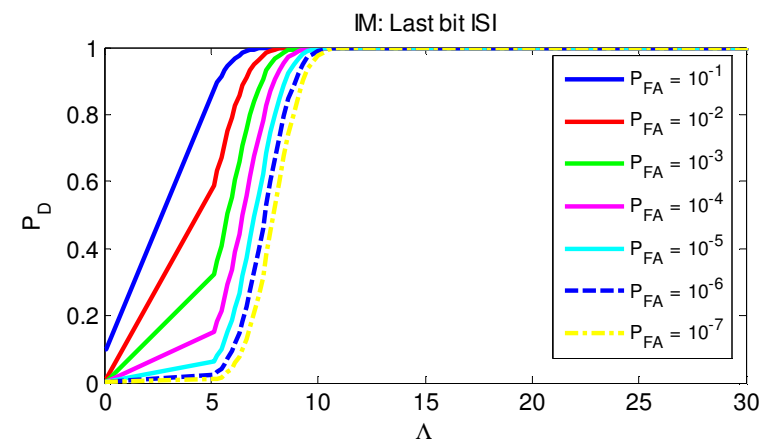

(a)

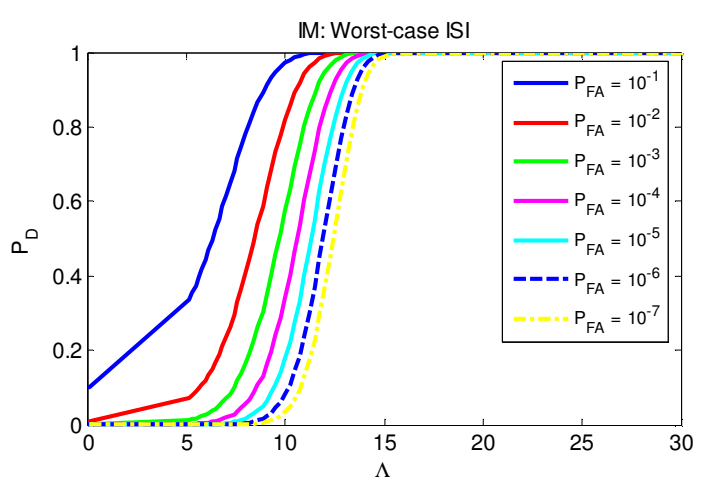

(c) communication in nanonetworks," Nano Communication Networks, vol. 1, pp. 289-300, Elsevier, 2010.

[2] T. Nakano, A. W. Eckford, and T. Haraguchi, Molecular Communication, Cambridge University Press, 2013.

[3] M. U. Mahfuz, D. Makrakis, and H. T. Mouftah, "A Generalized Strength-Based Signal Detection Model for Concentration-Encoded Molecular Communication," in Proc. BodyNets 2013, Boston, MA, USA, 2013, pp. 461-467.

[4] M. J. Moore and T. Nakano, "Synchronization of Inhibitory Molecular Spike Oscillators," in BIONETICS-2011, York, UK.

[5] H. C. Berg, Random Walks in Biology, Princeton University Press, NJ, USA, 1993.

[6] M. U. Mahfuz, D. Makrakis, and H. T. Mouftah, "A Comprehensive Study of Sampling-Based Optimum Signal Detection in Concentration-Encoded Molecular Communication," in IEEE Transactions on NanoBioscience, Vol. 13, Issue 3, pp. 208-222, September, 2014.

[7] T. S. Rappaport, Wireless communications : principles and practice. Upper Saddle River, Prentice Hall PTR, USA, 2002.

[8] S. M. Kay, Fundamentals of statistical signal processing, Vol. 2 Detection Theory, Prentice-Hall PTR, NJ, USA, 1993.

[9] M. U. Mahfuz, D. Makrakis, and H. T. Mouftah, "A Comprehensive Analysis of Strength-Based Optimum Signal Detection in Concentration-Encoded Molecular Communication With Spike Transmission," in IEEE Transactions on Nanbioscience, Vol. 14, No. 1, pp. 67-83, January 2015.

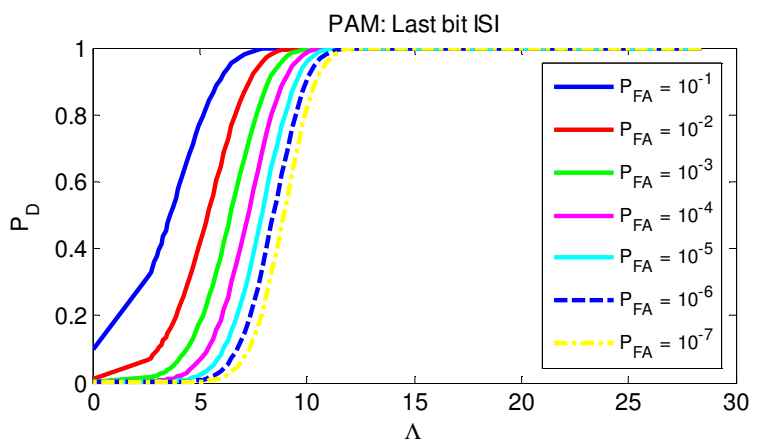

(b)

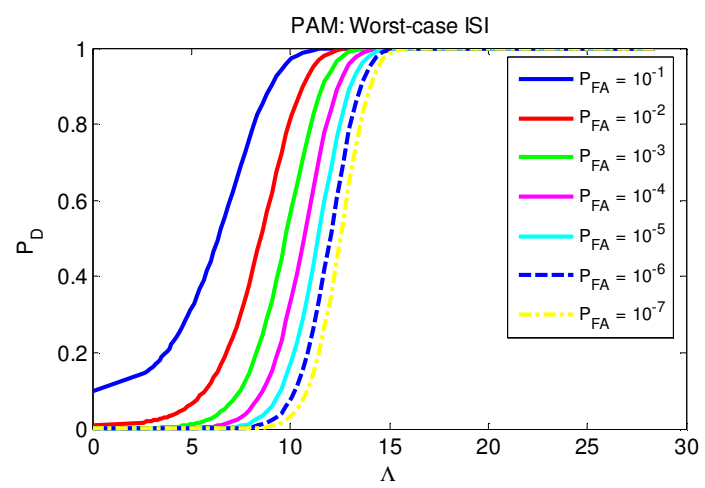

(d)

Fig. 4. Detection probability versus SNR characteristics in the presence of ISI. 